\title{
A PROPÓSITO DE LA MUERTE
}

\author{
Manuel Márquez Garrido* \\ José ARENAS FernándeZ ** \\ Diego J. Feria Lorenzo** \\ Rocío LeÓn LóPEZ ${ }^{* *}$ \\ ANa BARquero Gonzalez* \\ M. ${ }^{a}$ Carmen Carrasco* \\ ana CARMen Macias Rodríguez*
}

\section{RESUMEN}

El sentido del siguiente artículo es dar a conocer una lectura familiar para muchos profesionales de los Cuidados y nueva para otros, a veces la intuición nos ha llevado a un cuidar mejor, apareciendo conceptos profesionales como: confianza, empatía, autonomía, cuidados y esperanza, que nos capacitan cada vez más para una relación terapéutica de calidad.

Valoramos, como muy importante, conocer las distintas fases psicológicas por las que pasa el paciente terminal.

Palabras clave: cuidados paliativos, interrelación paciente-enfermera.

\section{SPEAKING ABOUTH THE DEATH}

\section{SUMMARY}

The sense of the following article is to present a familiar reading for many professionals of cares and new for others, sometimes the intuition has taken us to improve the cares, appearing professional concepts as confidence, empathy, autonomy, cares and hope, that enable to us more and more for a therapeutic relation of quality. We valued, as very important, to know the psychological different phases through which passes the terminal patient.

Keywords: palliative cares, patient-nurse interrelation "...si la muerte pisa mi huerto, quién firmará que he muerio de muerte natural, quièn será el buen amigo que morirá conmigo aunque sea un tanto así,..."

J. M. SERRAT

\section{INTRODUCCIÓN}

Los profesionales de Enfermería experimentan el mismo duelo que las demás personas cuando se enfrentan con una pérdida.

Nuestra profesión lleva implícito el contacto con el dolor, la muerte, la alegría, la recuperación y todos los estadios intermedios.

A diario existen enfermeros que desempeñan su labor al pie de pacientes moribundos, por lo que se establecen una serie de relaciones y de lazos mutuos. El objetivo principal, es la atención y prestación de cuidados al paciente desde un punto de vista terapéutico, siendo conscientes del aumento de relación existente entre ambos, de ésta manera al llegar el desenlace es normal que los enfermeros pasen por una etapa de duelo, denominado duelo de los cuidadores.

Existen profesionales que se afectan de tal manera con la pérdida sufrida, que les incapacita terapéuticamente, olvidando la importancia que tiene el apoyo que tiene que prestar a los familiares del fallecido. Por tanto nos encontramos ante un tema de equilibrio y madurez emocional, requisitos necesarios en todo profesional que se precie. No debemos olvidar encontrar el modo de renovar la propia energía. El día que aceptemos las actitu- 
des y sentimientos sobre las pérdidas y el duelo, nos encontraremos preparados para llevar a cabo las intervenciones terapéuticas que los demás demandan.

Abordaremos en el presente artículo la relación terapéutica que se establece entre el profesional enfermero y el paciente desde los conceptos de confianza, empatía, autonomía, cuidado y esperan$z a$, sin olvidar nunca la aceptación de las distintas fases psicológicas por las que pasa todo paciente terminal, así como sus necesidades elementales.

\section{LA ENFERMEDAD TERMINAL}

Una enfermedad terminal es aquella cuyo resultado final es la muerte.

El diagnóstico de enfermedad terminal, tal vez sea uno de los retos más difíciles que debe afrontar un individuo en su vida, especialmente cuando afecta en etapas tempranas de la vida.

Como figura en el Código Deontológico, el objetivo de la atención a las personas en situación de enfermedad terminal, no es acortar ni alargar su vida, sino promover su máxima calidad posible. El tratamiento de la situación de agonía debe adaptarse a los objetivos de confort, sin pretender alargar innecesariamente ni acortar deliberadamente. De entre las patologías cuya visión final puede ser la muerte, nuestra sociedad vive con sentimiento de duelo a lo largo de todo el proceso las neoplasias y el VIH/SIDA.

La forma en que una persona se prepara y enfrenta a la muerte va a depender de dos factores: -El significado de la misma.

- Los mecanismos de defensa utilizados durante toda la vida para tratar los problemas.

Para muchas personas su vida ha sido un continuo luchar y esforzarse por conseguir objetivos inalcanzables, mientras que otras aceptan la vida tal y como les viene. Por lo tanto van a afrontar su muerte de la misma manera que han afrontado su vida.

\section{MORIR CON DIGNIDAD}

Una vez agotados todos los recursos terapéuticos de que gozamos en la actualidad con los pacientes terminales van a sucederse una serie de periodos de tiempo en los que la enfermedad progresa y se estaciona de forma intermitente e irregular, apareciendo entonces problemas de todo tipo. Cuando el final se ve inmediato, los problemas aumentan cuantitativa y cualitativamente, no solo para el enfermo, sino también para los familiares y consecuentemente para el equipo terapéutico. El problema de la muerte va más allá de las interpretaciones teóricas o conceptuales para adentrarse en el terreno espiritual y de las emociones, por ello la muerte supone en muchos casos el enfrentamiento con el único fenómeno irremediable en la vida. De ahí, que ante ella, haya que abordar problemas de diferentes parcelas, como los relativos a aspectos psicológicos, sociales, culturales religiosos o morales.

De forma contraria al pensamiento general, una de las experiencias más gratificante de la Enfermería es la de ayudar a un ser humano a morir dignamente. Morir con dignidad, significa entre otras cosas, morir cada uno su propia muerte.

Tanto la persona moribunda como los que quedan vivos deben participar de manera plena y completa en su resolución. Cabe entender por lo tanto, que cuando la muerte es digna el paciente controla su propio destino, decide cuando abandonar los tratamientos agresivos, rechaza la última intervención quirúrgica, en resumen, es quién pone fin a las molestias de las terapias dolorosas. Se deduce pues, que en éste tipo de muerte, la paz, la serenidad y la aceptación van a sustituir a la negación, la lucha y el resentimiento. En una palabra, se opta por la calidad y no por la duración de la vida

La persona valora y aprecia cada nuevo día, pero se alegra de antemano del día en que acabará 
su sufrimiento. No teme a la muerte. Es la última etapa del crecimiento, que supone el término de una vida productiva y fructífera. (Houlberg, 1992)

\section{EL PROFESIONAL DE ENFERMERÍA ANTE EL ACTO DE LA MUERTE}

El personal de Enfermería proyecta sus propias actitudes, valores, creencias y prejuicios en la atención hacia los moribundos.

Si preguntásemos como consideran los cuidadores el acto de morir, las respuestas que obtendríamos serían: "muy doloroso", "desconcertante", "indiferente", "como una bendición", entre otras. Como presuponemos, estas actitudes se van a ver proyectadas fácilmente hacia el paciente terminal. De aquí la necesidad de una evaluación por parte de los profesionales con el fin de capacitarse para prestar una calidad de atención lo más objetiva posible.

En el currículum básico de Enfermería se capacita, más que suficientemente, a los futuros profesionales para detectar las necesidades biológicas o físicas del paciente moribundo. Cada vez son más, las escuelas que integran en sus planes de estudios asignaturas referentes al paciente terminal o cuidados paliativos.

Los profesionales de Enfermería que contribuyen a aliviar los síntomas físicos que conlleva la muerte, no viven ésta situación como amenaza, pero sí sienten generalmente angustias y muchas dudas al involucrarse en una relación terapéutica y significativa de apoyo al moribundo.

\section{EL DUELO DEL PERSONAL DE ENFERMERÍA}

Los cuidadores experimentan el mismo duelo que el resto de las personas cuando se enfrentan a una pérdida. Esta se puede producir a lo largo del ciclo vital de las personas.

El trato diario con pacientes moribundos, va a dar lugar al aumento de relaciones entre ambos, desarrollándose empatía y lazos mutuos, siendo siempre el objetivo el paciente y el profesional el sujeto terapeuta, es lógico esperar en éste último una aflicción, necesaria como vía de escape para su salud psicológica. Hay profesionales que se afligen tanto, que no pueden desarrollar la labor de apoyo, en el duelo, a los familiares y allegados.

Muchas instituciones tienen integrados equipos de apoyo para los profesionales de Enfermería que atienden a pacientes moribundos, que ayudan a resolver sus dulilos. Cuando los enfermeros descubran y admitan las propias actitudes y sensaciones sobre las pérdidas y el duelo, entonces estarán capacitados para llevar a cabo las intervenciones terapéuticas que los demás necesitan.

Es importante reseñar diversos modos de nutrir a los cuidadores para renovar la propia energía. Estos pueden fortalecerse de diversos modos: creando nuevas fuentes de confort, probando nuevas experiencias, dedicándose tiempo a sí mismo, haciendo deporte, viajando, montándose nuevas relaciones, practicando la meditación diaria, etc.

Podríamos recurrir al término insigth, como referencia a dos tipos de conocimientos, por un lado la sabiduría de conocerse a sí mismo y por otro, la visión clara para conocer a los demás. En la medida que los profesionales que tratan con pacientes moribundos vayan ganando en insigth va a aumentar posiblemente la capacidad para ver y comprender claramente la naturaleza de las cosas.

\section{LA RELACIÓN TERAPÉUTICA}

Las personas son seres sociales, viven en contacto permanente con otras personas, estableciéndose una relación interpersonal, que influyen en la cotidianeidad del individuo. Para Morrisson, (1999), la relación terapéutica es un intercambio de energía entre dos personas, un flujo que mueve al paciente a llevar una conducta más eficaz. La energía del enfermero se utiliza para dirigir al paciente hacia una forma más constructiva del 
pensamiento y una forma más eficaz para manejar sus problemas. El cuidador utiliza su caudal de energía dirigida hacia la salud para, en primer término, equilibrar o estabilizar a sus pacientes. Luego, ayuda al paciente a movilizar y dirigir su propia energía en una dirección más satisfactoria. $\mathrm{El}$ arte de la enfermería implica un intercambio de energía que se produce cada vez que el enfermero interactua con su paciente.

\section{DINÁMICA DE LA RELACIÓN TERAPÉUTICA CON EL PACIENTE TERMINAL}

Todo tipo de relación entre las personas incluye dinámicas, que como sabemos hacen referencia a las interacciones que se producen entre varias fuerzas.

La relación terapéutica se diferencia de otras relaciones, como la social o la laboral, en que el objetivo principal es el paciente y se establece de manera consciente.

Los elementos de la relación terapéutica incluye los conceptos de confianza, empatía, autonomía, cuidado y esperanza. Utilizar estos conceptos como soporte, ayuda al enfermero a fomentar la aptitud y sensibilidad necesaria para dirigir su energía hacia una relación de ayuda eficaz.

\section{CONFIANZA}

Según Anderson, Anderson y Glauze, (1994), la confianza se define como un proceso arriesgado en el cual la situación de un individuo depende de la conducta futura de otra persona. La falta de confianza lleva al aislamiento de las personas y a la falta de apoyo entre ellas.

Para Travelbee, (1971), la confianza es la convicción de que otros sujetos son capaces de ayudar en momentos de necesidad y que van a hacerlo.

Según estas definiciones, el interés del paciente será el móvil que establezca la base de una relación de confianza entre profesional - enfermo.
Cuando el paciente necesita ayuda, su energía está a un nivel muy bajo, dato que debe conocer el profesional de Enfermería.

Las distintas formas en que el personal de Enfermería canaliza su energía para entablar la confianza con el paciente son:

- Evaluar la capacidad del paciente para confiar en los demás. El enfermero debe dar muestras mediante sus distintas acciones para que el enfermo confíe cada vez más en él.

-El enfermero debe ser íntegro con sus pacientes. La honestidad debe ser una característica a destacar siempre.

- La comunicación clara establece un grado alto de confianza. Los tecnicismos sanitarios, el lenguaje falso, la verborrea, influyen de manera considerable en el grado de confianza del paciente hacia los profesionales. Así mismo es de suma importancia dejar hablar al paciente el tiempo que necesite y sobre todo que exprese sus sentimientos y temores. El cuidador ha de ser hábil en la comunicación no verbal.

\section{EMPATÍA}

Es la capacidad de sentir con el otro. El enfermero entra a formar parte de la vida de otra persona, compartiendo su mundo, sus emociones $y$ actitudes.

La relación de empatía se lleva a cabo mediante la comunicación verbal, la no verbal y con la conducta que establecemos.

Puesto que estamos hablando de pacientes terminales, destacaremos algunas técnicas de comunicación no verbal:

-Cinética: movimientos corporales de los cuales podemos deducir significados, como gestos, expresiones faciales, movimientos de brazos, etc.

-Contacto físico: es importantísimo utilizar el tacto con pacientes que estén en situación terminal, a veces va a ser el único vehículo de comunicación. 
-Proximidad: es la distancia o espacio entre las personas cuando se comunican, con éste tipo de pacientes es fundamental estar acostumbrados al espacio íntimo, hasta los $45 \mathrm{~cm}$.

-Silencio: a veces la mejor comunicación será guardar silencio acompañando a un paciente en estas situaciones. Es preciso que dominemos ésta técnica, son muchos los profesionales que se sienten fracasados si no han recibido habilidades y entrenamientos.

- Paralenguaje: en ella influye la calidad de la voz, el tono, volumen, dicción y forma de dar el mensaje.

Como describe Ann Isaacs (1998), en Enfermería en Salud Mental y Psiquiátrica, las conductas empáticas más comunes que podemos utilizar son: - Centrarse en los sentimientos del paciente.

- Hacer preguntas abiertas.

- Usar tono de voz cálido.

- Transmitir una actitud no enjuiciadora.

- Mantener el contacto visual.

-Asentir periódicamente con la cabeza.

-Sonreir frecuentemente.

- Gesticular suavemente.

- Abrir los brazos.

- Inclinarse ligeramente hacia delante.

- Demostrar comodidad.

- Sincronizar los movimientos con los del paciente.

\section{AUTONOMÍA}

La autonomía guarda una relación directa con la actitud para dirigir y controlar las propias actividades y el propio destino.

El paciente ingresado y envuelto por el mundo sanitario, pierde su capacidad para poder decidir y tomar decisiones que le afecten.

Muchos profesionales de Enfermería caen en el error de ser paternalistas y no dejan opción al paciente de resolver situaciones propias, cayendo en una superprotección.
Una tendencia actualizada de los cuidados nos llevaría a que el paciente reestableciera su autonomía lo antes posible siendo partícipe en las disposiciones que le atañan.

\section{CUIDADOS}

El ser humano necesita ser amado. Según la pirámide de Necesidades de A. Maslow, el amor se sitúa en el tercer nivel.

El cuidado es la columna vertebral de la Relación Terapéutica, es la energía que otorga al enfermero la capacidad de aceptar absolutamente a todos los pacientes, conectemos o no con ellos.

La base profesional asistencial de la Enfermería, es la prestación de cuidados, una profesión que fundamenta su ser en ésta tarea. El arte de prestar cuidados se lleva a cabo, en la realización de cada acto, cada vez que efectuamos un movimiento, un ademán y cada vez que nos relacionamos con el paciente. Cuidar es una energía capaz de transmitir duda, emotividad y sentimiento.

Saber cuidar, es imprescindible para una buena relación terapéutica.

\section{ESPERANZA}

Para muchas personas la esperanza va relacionada con el futuro. Esperanza quiere decir expectación, el que espera con ansia. Para Dufault y Martocchio (1985), es la fuerza vital dinámica, multidimensional, caracterizada por una expectativa confiada, aunque incierta, de alcanzar un buen futuro.

Podemos definirla también, como la energía que motiva hacia la consecución de un buen estado de salud. Cuando nos planteamos una perspectiva a cierto plazo, debe descansar en el pilar del realismo, siendo posible su consecución, al menos en gran parte. Lo contrario, la expectativa negativa, puede retrasar la normalidad en la persona,

Para el personal de Enfermería, la esperanza es la herramienta de energía terapéutica que va a 
influir de manera notable en el resultado final de los cuidados. Desde la Enfermería, nunca deben crearse expectativas falsas al paciente terminal.

\section{NECESIDADES DE LOS MORIBUNDOS}

Para la mayoría de pacientes moribundos, se presentan una serie de necesidades especiales en sus últimos días. La jerarquía de los pacientes moribundos, Ebersole y Hess, (1994), se constituye en una pirámide que tendría en su primer nivel: obtener alivio de los síntomas físicos, conservar la energía y estar sin dolor. A continuación, en el nivel superior, el paciente tendría la oportunidad de expresar miedos ocultos, así como confiar en sus cuidadores, sentir en todo momento que se le dice la verdad y estar seguro. En el tercer nivel, hablar y ser escuchado con comprensión, ser querido y compartir amor y estar con personas cariñosas cuando llegue su muerte. En el cuarto nivel: mantener el respeto ante la debilidad progresiva, mantener la independencia, sentimiento de ser una persona normal con el derecho vital a tener fin, conservando su identidad personal. En el nivel más alto se encontraría la aceptación del futuro inevitable, compartiéndolo, así como percibir el significado de la muerte.

\section{OTRAS REFLEXIONES}

Además de los elementos citados en la relación terapeútica hay aspectos éticos que no debemos olvidar en la práctica diaria:

- El respeto a la confidencialidad.

-El respeto a la individualidad, facilitando y a veces incitando al diálogo.

-El derecho a la información que tiene, tanto el paciente como la familia.
-El respeto a las últimas voluntades (cuando el paciente pierde la capacidad de decidir):

- El tratamiento que desearía recibir.

-A quién otorga los poderes (especificar qué persona).

-El derecho al consentimiento informado que tiene tanto el paciente como la familia.

-El derecho de la no maleficencia.

-El respeto al principio de autonomía.

-El derecho a la justicia.

No se debe olvidar que el papel de enfermería es clave, ya que suele ser el profesional más cercano al paciente y a la familia y asi lo perciben, por tanto el que puede en mayor grado canalizar su ansiedad proporcionándole información y dando su apoyo en esos momentos tan necesarios.

\section{BIBLIOGRAFIA}

Bermejo, J.C. "Relación de ayuda y Enfermería". Cuadernos del Centro de Humanización de la Salud. Edit. Religiosos Camilos. 4. ${ }^{a}$ Edición. Madrid. 1996.

Bermejo, J.C.; Carabias, R. "Relación de ayuda y Enfermería". Cuadernos del Centro de Humanización de la Salud. Edit. Religiosos Camilos. Madrid. 1996.

Bermejo, J.C. "Apuntes de relación de ayuda". Cuadernos del Centro de Humanización de la Salud. Edit. Religiosos Camilos. 4. ${ }^{a}$ Edición. Madrid. 1996.

Gomez, M. "Cuidados Paliativos e intervención psicosocial en enfermos terminales". Edit. Instituto Canario de Estudios y Promoción Social y Sanitaria. Las Palmas. 1994.

KüBler, R. "Morir es de vital importancia". Edit. Luciérnaga. Barcelona 1995.

Lara, A.; Zamora, H.; Esquivel, V.M.; "Cuidados paliativos: la alternativa para una mejor fase terminal". Rev. Hospital General Dr. Gea González. Vol. 2. N. ${ }^{\circ}$ 1. Enero-Marzo 1999.

MORRISON, M. "Fundamentos de Enfermería en Salud Mental": Edit. Mosby. Madrid. 1999.

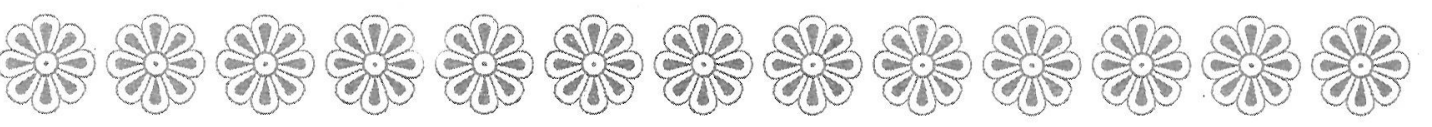

